\title{
The General Form of Linearized Exact Solution for the KdV Equation by the Simplest Equation Method
}

\author{
Sen-Yung Lee ${ }^{1}$, Chun-Ku Kuo ${ }^{1,2, *}$ \\ ${ }^{1}$ Department of Mechanical Engineering, National Cheng Kung University, Taiwan, R.O.C. \\ ${ }^{2}$ Department of Mechanical Engineering, Air Force Institute of Technology, Taiwan, R.O.C.
}

Email address:

lon12110213@yahoo.com.tw (Chun-Ku K.)

\section{To cite this article:}

Sen-Yung Lee, Chun-Ku Kuo. The General Form of Linearized Exact Solution for the KdV Equation by the Simplest Equation Method. Applied and Computational Mathematics. Vol. 4, No. 5, 2015, pp. 335-341. doi: 10.11648/j.acm.20150405.11

\begin{abstract}
The general form of linearized exact solution for the Korteweg and de Vries (KdV) equation, with an arbitrary nonlinear coefficient, is derived by the simplest equation method with the Bernoulli equation as the simplest equation. It is shown that the proposed exact solution overcomes the long existing problem of discontinuity and can be successfully reduced to linearity, while the nonlinear term coefficient approaches zero. Comparison of four different soliton solutions is presented. A new phenomenon, named soliton sliding, is observed.
\end{abstract}

Keywords: General Form, Linearized, KdV, Simplest Equation Method, Bernoulli, Discontinuity, Soliton Sliding

\section{Introduction}

The word soliton was first used in Zabusky and Kruskal's paper in 1965 [1]. A soliton can be defined as a solution to a nonlinear partial differential equation that exhibits the following three properties: (i) the solution should demonstrate a permanent form wave; (ii) the solution is localized, which means that the solution either decays exponentially to zero, or converges to a constant at infinity; and, (iii) the soliton interacts with other solitons preserving its character. The Korteweg and de Vries $(\mathrm{KdV})$ equation is a typical nonlinear partial differential equation that provides soliton solutions.

The $\mathrm{KdV}$ equation [2] in dimensionless variables is expressed as

$$
u_{t}+\alpha u u_{x}+u_{x x x}=0
$$

where $\alpha$ is scaled to any real number [3]. This nonlinear partial differential equation was derived by Kortewege and de Vries to describe shallow water waves of long wavelength and small amplitude. It is the simplest nonlinear dispersive equation embodying two effects: the nonlinear term, $u u_{x}$, and the linear dispersion term, $u_{x x x}$. The nonlinearity of $u u_{x}$ tends to localize the wave, whereas dispersion spreads the wave out. The delicate balance between $u u_{x}$ and $u_{x x x}$ defines the formulation of solitons that consist of single humped waves. In addition, there are various physical systems that can also be modeled by the $\mathrm{KdV}$ equation, such as acoustic waves in harmonic crystals and ion waves in plasmas [4].

Exact solutions of the $\mathrm{KdV}$ equation with a variable nonlinear term coefficient have been developed through a variety of analytical techniques, such as tanh-coth method [5-7], sine-cosine method [5], Hirota's direct method [3, 8-9], and Exp-function method [10], among others [11-13].

From the literature $[3,5,7-10]$, it can be found that all exact solutions of the $\mathrm{KdV}$ equation will approach to infinity and do not satisfy the continuity condition when the nonlinear term coefficient is zero. Obviously, they can not be reducible to linear solutions.

In this paper, the linearized exact solutions of the $\mathrm{KdV}$ equation are derived by the simplest equation method with the Bernoulli equation as the simplest equation. It is shown that the proposed exact solutions overcome the problem of discontinuity and can be successfully reduced to linear ones, while the nonlinear term coefficient of the differential equation approaches zero. Differences among existing and linearized solutions are elaborated. Several numerical results are illustrated, through which a new phenomenon is revealed.

\section{Existing Exact Solutions of the KdV Equation}

Exact solutions of the $\mathrm{KdV}$ equation with a variable 
nonlinear term coefficient developed by the tanh-coth method [5-7], the sine-cosine method [5], the Exp-function method [10] and others [11-13] can be summarized as

$$
\begin{gathered}
u_{1}=\frac{3 c}{\alpha} \sec h^{2}\left[\frac{\sqrt{c}}{2}(x-c t)\right], c>0 \\
u_{2}=\frac{-3 c}{\alpha} \csc h^{2}\left[\frac{\sqrt{c}}{2}(x-c t)\right], c>0 \\
u_{3}=\frac{-c}{\alpha}\left(1+3 \cot ^{2}\left[\frac{\sqrt{c}}{2}(x-c t)\right]\right), c>0 \\
u_{4}=\frac{-c}{\alpha}\left(1+3 \tan ^{2}\left[\frac{\sqrt{c}}{2}(x-c t)\right]\right), c>0
\end{gathered}
$$

and

$$
\begin{gathered}
u_{5}=\frac{3 c}{\alpha} \sec ^{2}\left[\frac{\sqrt{-c}}{2}(x-c t)\right], \quad c<0 \\
u_{6}=\frac{3 c}{\alpha} \csc ^{2}\left[\frac{\sqrt{-c}}{2}(x-c t)\right], \quad c<0 \\
u_{7}=\frac{-c}{\alpha}\left(1-3 \operatorname{coth}^{2}\left[\frac{\sqrt{-c}}{2}(x-c t)\right]\right), \quad c<0 \\
u_{8}=\frac{-c}{\alpha}\left(1-3 \tanh ^{2}\left[\frac{\sqrt{-c}}{2}(x-c t)\right]\right), \quad c<0
\end{gathered}
$$

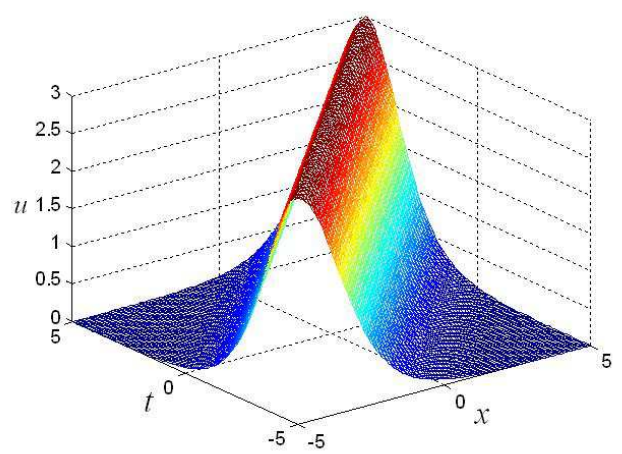

(a)

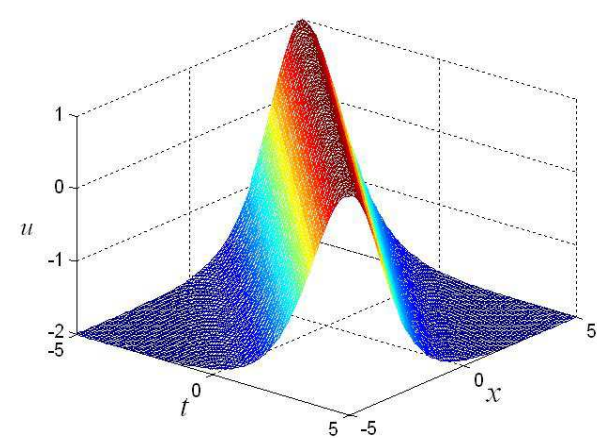

(b)

Figure 1. Existing soliton solutions. [ $\alpha=1$; (a) equation (2.1) with $c=1$, (b) equation (2.8) with $c=-1$. where $\mathrm{c}$ is the wave speed. It is noted that equations (2.1-8) can all be obtained by the tanh-coth method [3], while equations (2.1-2) and (2.5) can also be found by the sine-cosine method [3]. From the solution forms, it can be found that among them, only solutions $u_{1}$ and $u_{8}$ are soliton solutions, as shown in Figure 1.

When the nonlinear term coefficient in equation (1.1) is specified as 6 , the exact single-soliton solution was obtained by using Hirota's transformation [8], $\alpha u=12(\ln F)_{x x}$, as

$$
u_{9}=\frac{2 k^{2} e^{k\left(x-k^{2} t\right)}}{\left(1+e^{k\left(x-k^{2} t\right)}\right)^{2}}
$$

The two-soliton and the N-soliton solutions can also be obtained by the same transformation [3,9]. Here the function $F(x, t)$ is assumed to have a perturbation expansion form; $k$ is determined to be an arbitrary constant; $\mathrm{N}$ is a positive integer. Setting $k=\sqrt{c}$ in equation (2.9) yields a result that is the same as the solution, $u_{1}$, obtained by tanh-coth and sine-cosine methods.

It is well known that, for solution continuity, if the nonlinear term coefficient in nonlinear differential equations (1.1) approaches zero, the equation could be reduced to a linear one, and the nonlinear solutions should be reducible to linear solutions. However, from the existing solutions above, it can be observed that all solutions of the $\mathrm{KdV}$ equation are proportional to $1 / \alpha$. When the nonlinear term coefficient, $\alpha$, is reduced to zero, the solutions become infinity; therefore, none will satisfy the continuity condition at $\alpha=0$. In the following, new exact solutions of the $\mathrm{KdV}$ equation with linearized solutions are developed.

\section{Linearized Solutions for the KdV Equation}

Assume a partial differential equation. After a transformation by the wave variable $\xi=x-c t$, a nonlinear ordinary differential equation (ODE) results.

$$
P\left(U, U_{\xi}, U_{\xi \xi}, \ldots\right)=0
$$

\subsection{The Simplest Equation Method}

The simplest equation method [14-20] is a method commonly used to develop the exact solutions of some ordinary nonlinear differential equations. Here, the method is applied to develop exact solutions of the $\mathrm{KdV}$ equation.

For a large class of equations of the type represented in (3.1), the exact solution can be assumed to be in the form of

$$
U(\xi)=\sum_{i=0}^{L} a_{i}[Y(\xi)]^{i}
$$

where $L>0$ and must be an integer; $a_{i}$ are parameters; 
$Y(\xi)$ is a solution of a certain nonlinear ordinary differential equation with an exact solution, referred to as the simplest equation. $L$ is determined by substituting equation (3.2) into equation (3.1) and balancing the linear term of the derivative's highest order with the highest nonlinear term in equation (3.1).

It is known that the Riccati $[21,22]$ equation is commonly selected as the simplest equation for the simplest equation method. However, Kudryashov [20] pointed out that the solution method is equivalent to tanh-coth method, and so the derived exact solutions of the $\mathrm{KdV}$ equation will be the same as those given in equations (2.1-8).

In this paper, the Bernoulli equation was chosen as the simplest equation for the simplest equation method. The Bernoulli equation is in the form

$$
Y_{\xi}=a Y+b Y^{2}
$$

where $a$ and $b$ are constants. The exact solution of the equation above is [23]

$$
Y=\frac{a}{e^{-a\left(\xi+\xi_{0}\right)}-b}
$$

where $\xi_{0}$ is an integral constant, here set as $\xi_{0}=0$.

After substituting equations (3.2-3) into (3.1), and equating the coefficients of the same powers of $Y$ to zero in the result equation, a system of algebraic equations involving $a_{i},(i=0, \ldots, L)$ are derived. Having determined these parameters and using equation (3.4) an analytically closed form solution can be obtained.

\subsection{Linearized Solutions}

After a transformation by the wave variable $\xi=x-c t$, the $\mathrm{KdV}$ equation (1.1) can be transformed into the following nonlinear ordinary differential equation

$$
-c U+\frac{\alpha}{2} U^{2}+U \xi \xi=0
$$

Substituting equation (3.2) into equation (3.5) and balancing the linear term of the derivative's highest order, the balanced equation gives $L=2$. Therefore, the solution can be constructed as

$$
U(\xi)=a_{0}+a_{1} Y+a_{2} Y^{2}
$$

Substituting equations (3.3) and (3.6) into (3.5), and setting like power coefficients of $Y$ to zero, leads to a system of nonlinear relationships among the parameters of the solution and the parameters of the solved equation class.

$$
\begin{gathered}
Y^{0}:-c a_{0}+\frac{\alpha}{2} a_{0}^{2}=0 \\
Y^{1}:-c a_{1}+\alpha a_{0} a_{1}+a_{1} a^{2}=0 \\
Y^{2}:-c a_{2}+\alpha a_{0} a_{2}+\frac{\alpha}{2} a_{1}^{2}+3 a_{1} a b+4 a_{2} a^{2}=0 \\
Y^{3}: \alpha a_{1} a_{2}+2 a_{1} b^{2}+10 a_{2} a b=0 \\
Y^{4}: \frac{\alpha}{2} a_{2}^{2}+6 a_{2} b^{2}=0
\end{gathered}
$$

Solving equations (3.7-11) yields the following four cases

Case1. $a=\sqrt{c}, b=\alpha, a_{0}=0, a_{1}=-12 \sqrt{c}, a_{2}=-12 \alpha$,

Case2. $a=-\sqrt{c}, b=\alpha, a_{0}=0, a_{1}=12 \sqrt{c}, a_{2}=-12 \alpha$,

Case3. $a=\sqrt{c}, b=-\alpha, a_{0}=0, a_{1}=12 \sqrt{c}, a_{2}=-12 \alpha$,

Case4. $a=-\sqrt{c}, b=-\alpha, a_{0}=0, a_{1}=-12 \sqrt{c}, a_{2}=-12 \alpha$.

As a result, the four linearized exact solutions are derived as

$$
\begin{gathered}
u_{10}=\frac{-12 c e^{-\sqrt{c}(x-c t)}}{\left(e^{-\sqrt{c}(x-c t)}-\alpha\right)^{2}}, \\
u_{11}=\frac{-12 c e^{\sqrt{c}(x-c t)}}{\left(e^{\sqrt{c}(x-c t)}-\alpha\right)^{2}} \\
u_{12}=\frac{12 c e^{-\sqrt{c}(x-c t)}}{\left(e^{-\sqrt{c}(x-c t)}+\alpha\right)^{2}}, \\
u_{13}=\frac{12 c e^{\sqrt{c}(x-c t)}}{\left(e^{\sqrt{c}(x-c t)}+\alpha\right)^{2}}
\end{gathered}
$$

\begin{tabular}{|c|c|c|c|c|c|c|c|c|}
\hline \multicolumn{2}{|c|}{ Parameters } & \multicolumn{7}{|c|}{ Exact solutions } \\
\hline \multirow{2}{*}{$\alpha$} & \multirow{2}{*}{$c$} & \multicolumn{3}{|c|}{ Existing solutions } & \multicolumn{4}{|c|}{ Linearized solutions } \\
\hline & & Eq. (2.1) & Eq. (2.8) & Eq. (2.9) & Eq. (3.12) & Eq. (3.13) & Eq. (3.14) & Eq. (3.15) \\
\hline$>0$ & $>0$ & soliton & NA & soliton & Traveling wave & Traveling wave & soliton & soliton \\
\hline$>0$ & $<0$ & NA & soliton & soliton & Traveling wave & Traveling wave & Traveling wave & Traveling wave \\
\hline$<0$ & $<0$ & NA & soliton & soliton & Traveling wave & Traveling wave & Traveling wave & Traveling wave \\
\hline
\end{tabular}

When $c>0$, and for property (ii) mentioned in section 1 , as $\mathrm{x}$ approaches positive or negative infinity, the corresponding equations (3.12-15) all agree, equating to zero. Moreover, after numerical calculation using MATLAB, it can be seen that equations (3.12-13) with negative $\alpha$, and also equations (3.14-15) with positive $\alpha$, agree with property (i), demonstrating a wave of permanent form. The wave-type of the existing solutions, namely equations (2.1) and (2.8-9), as well as the linearized solutions, equations (3.12-15), are summarized in Table 1.

Table 1. Wave types of the existing and linearized solutions for the KdV equation. 


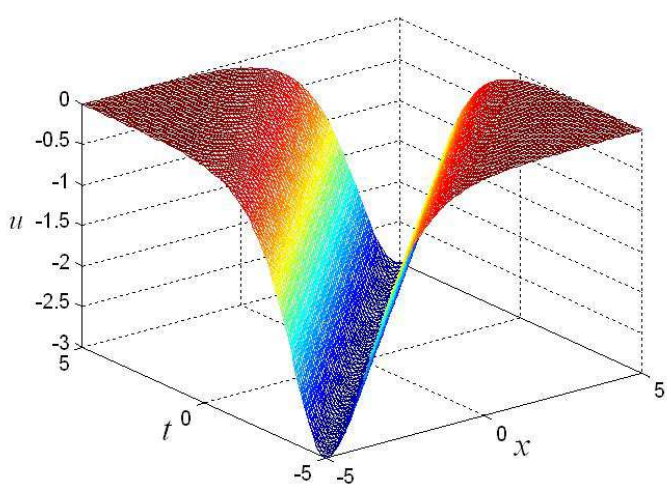

(a)

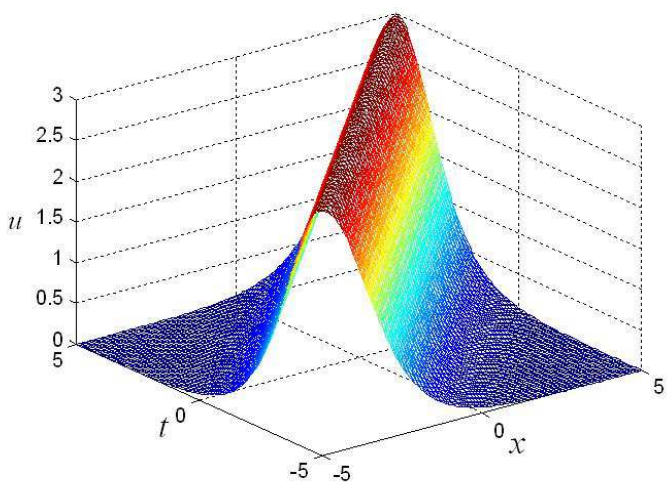

(b)

Figure 2. Linearized soliton solutions. [ $c=1$; (a) equation (3.12) with $\alpha=-1$, (b) equation (3.14) with $\alpha=1$.]

Moreover, observing the form of equations (3.12-15), it can be found that equation (3.12) will equal (3.13), and equation (3.14) will equal (3.15) as $\alpha= \pm 1$. These soliton solutions are shown in Figure 2. It is noted that equation (3.12) with $\alpha=-1$, and equation (3.14) with $\alpha=1$ could be equivalent to equation (2.1). This means, under certain conditions, that equations (3.12-13) and (3.14-15) are the same as (2.1). And for the continuous case, the soliton solution could be presented by equations (3.12-15) instead of equation (2.1). The evidence for this is found in Figures 1-2. Obviously, Figure 1a is the same as Figure 2b; and Figure 1a would be the same as Figure $2 \mathrm{a}$ if the $\alpha$ 's sign were inverted.

The aim of this paper is to emphasize that these general form solutions, equations (3.12-15), are linearized solutions. They are reducible to linear solutions just as nonlinear equation (1.1) can be reduced to a linear equation by limiting $\alpha$ to zero. In searching the associated literature, the above linearized solutions, which satisfy the physical continuity meaning for the $\mathrm{KdV}$ equation with a variable coefficient, are described for the first time. To demonstrate this claim, two numerical results are presented and illustrated in the next section.

\section{Numerical Results and Discussions}

As stated before, the $\mathrm{KdV}$ equation $[4,24]$ is the pioneering model for analytical soliton solutions. In order to present the soliton feature, we use the existing soliton solutions (equations (2.1) and (2.8)) and the linearized ones (equations (3.14-15)) for comparison with variable parameters $c$ and $\alpha$. First, for the four soliton solutions, we suggest that the wave speed $c$ affects the steepening of the solitary wave. And, it can be seen that a higher speed moves the wave location forward, with $t=2$ and $\alpha=6$, as shown in Figures 3-6. Second, the nonlinear term coefficient $\alpha$ makes two great differences between the existing soliton solutions and the linearized ones.

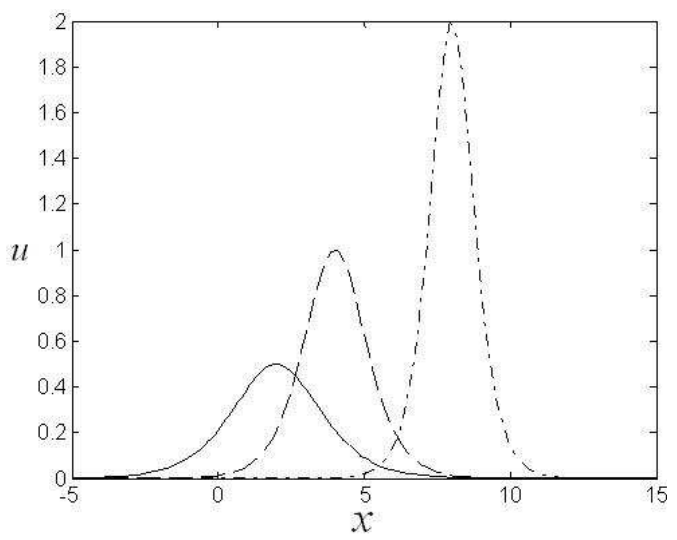

Figure 3. Influence of the wave speed $c$ on equation (2.1). [ $t=2, \alpha=6$; $c=1 ;--=: c=2 ;-:-: c=4$.

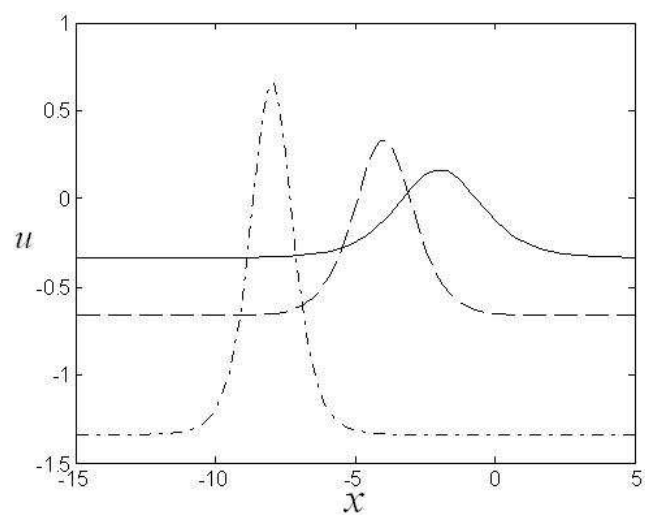

Figure 4. Influence of the wave speed $c$ on equation (2.8). [ $t=2, \alpha=6$; $c=-1 ;-=-: c=-2 ;-\div-: c=-4$.]

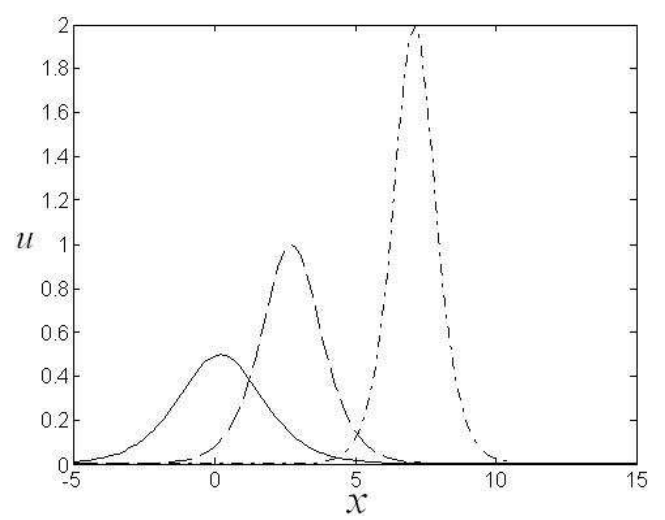

Figure 5. Influence of the wave speed $c$ on equation (3.14). [ $t=2$, $\alpha=6$; $c=1 ;-=-1 c=2 ;-1=4 c=4$ 


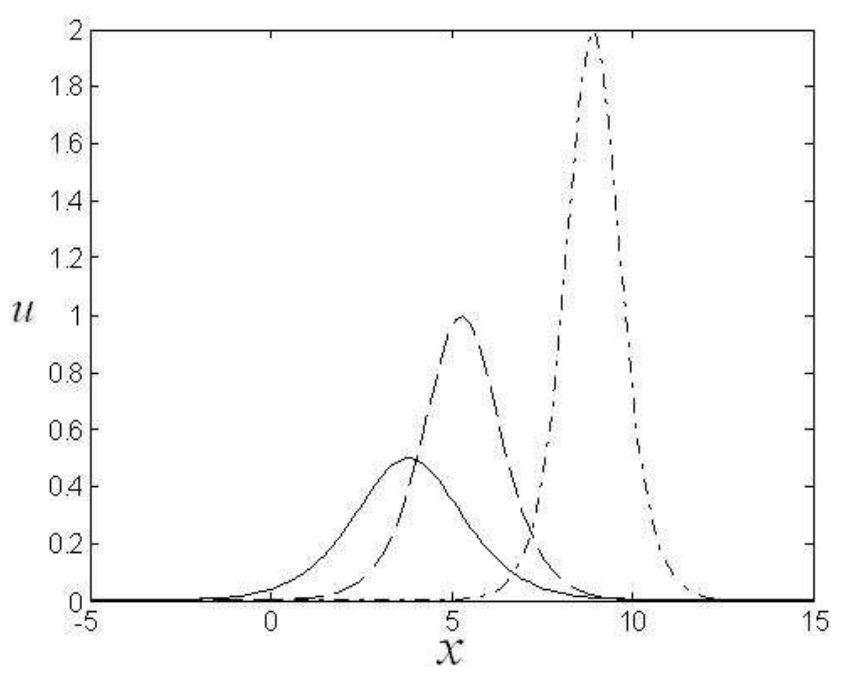

Figure 6. Influence of the wave speed $c$ on equation (3.15). [ $t=2$, $\alpha=6 ; \longrightarrow: c=1 ;-=-: c=2 ;-1=\because c=4$.

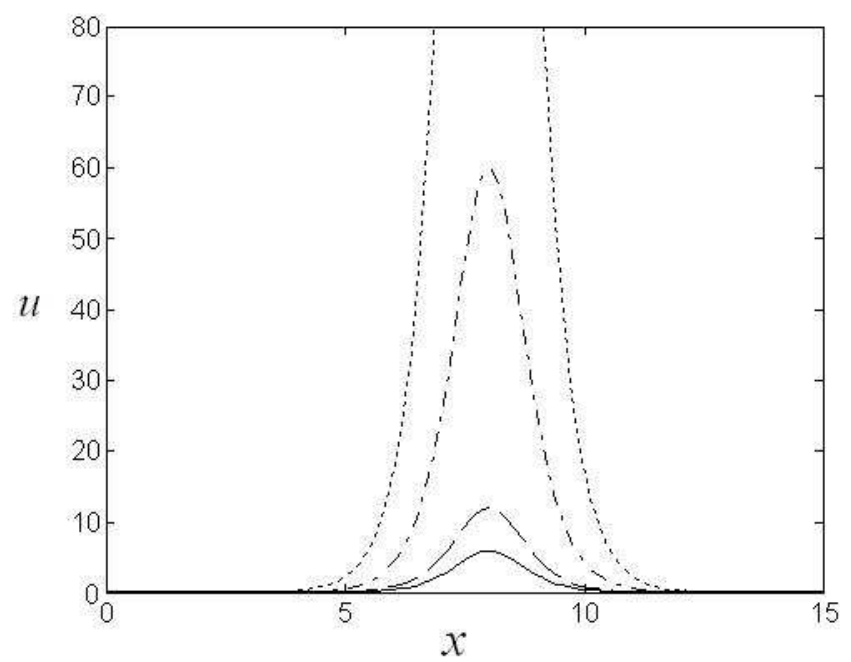

Figure 7. Influence of the nonlinear term coefficient $\alpha$ on equation (2.1). $[t=2, c=4$ แини: $\alpha=0.05$.]

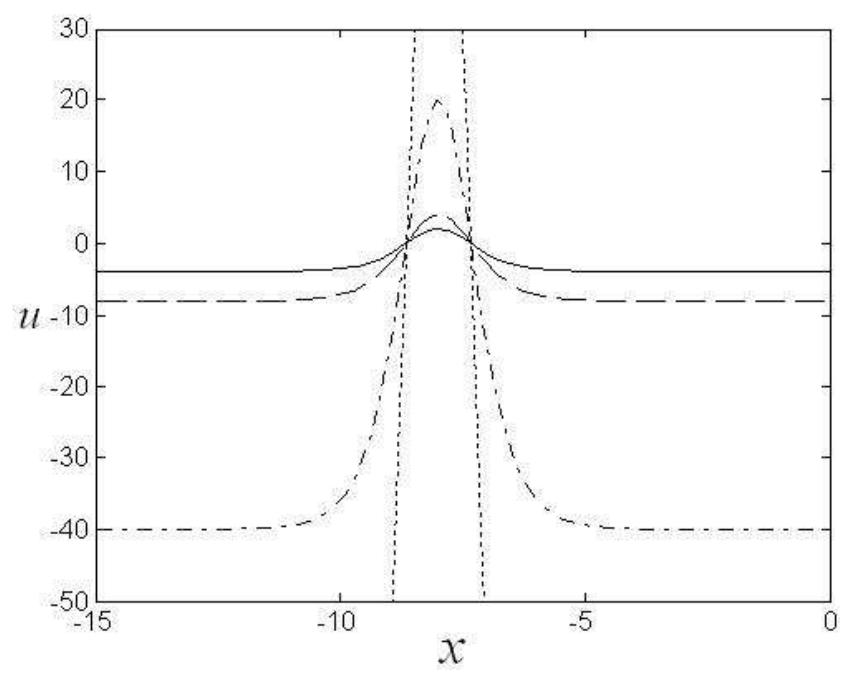

Figure 8. Influence of the nonlinear term coefficient $\alpha$ on equation (2.8). $[t=2, c=-4 ; \quad: \alpha=2 ;--=: \alpha=1 ;-\cdots=0.2$; ниини: $\alpha=0.05$.]

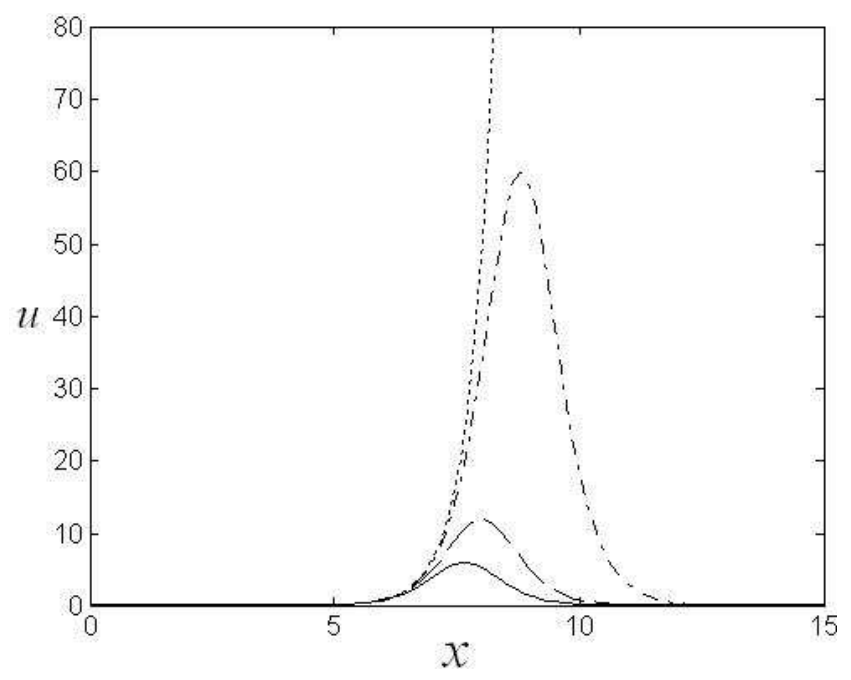

Figure 9. Influence of the nonlinear term coefficient $\alpha$ on equation (3.14). $[t=2, c=4 ;-: \alpha=2 ;--=: \alpha=1 ;--: \alpha=0.2$; ……: $\alpha=0$.

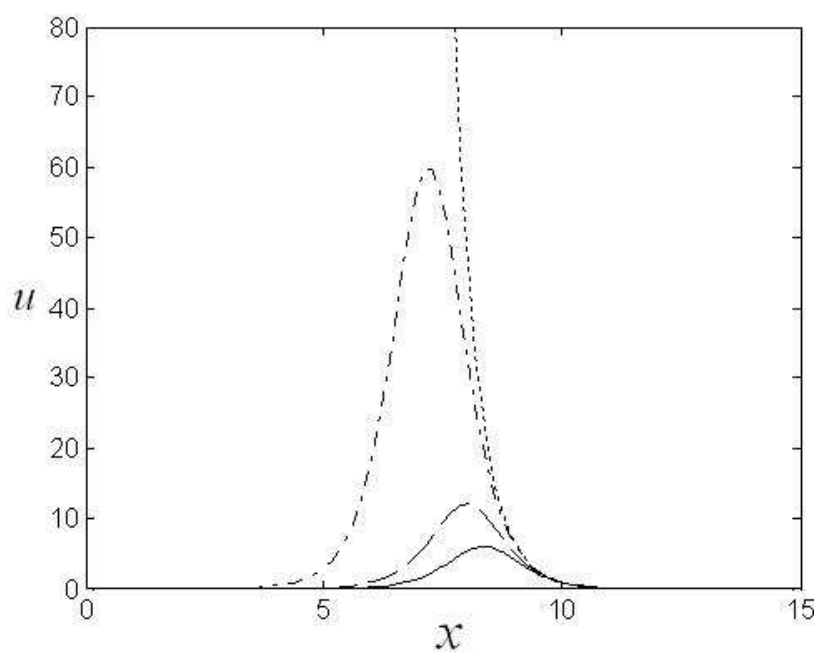

Figure 10. Influence of the nonlinear term coefficient $\alpha$ on equation (3.15). $[t=2, c=4$; $: \alpha=2 ;-=-: \alpha=1 ;=-: \alpha=0.2$; пимим! $\alpha=0$.

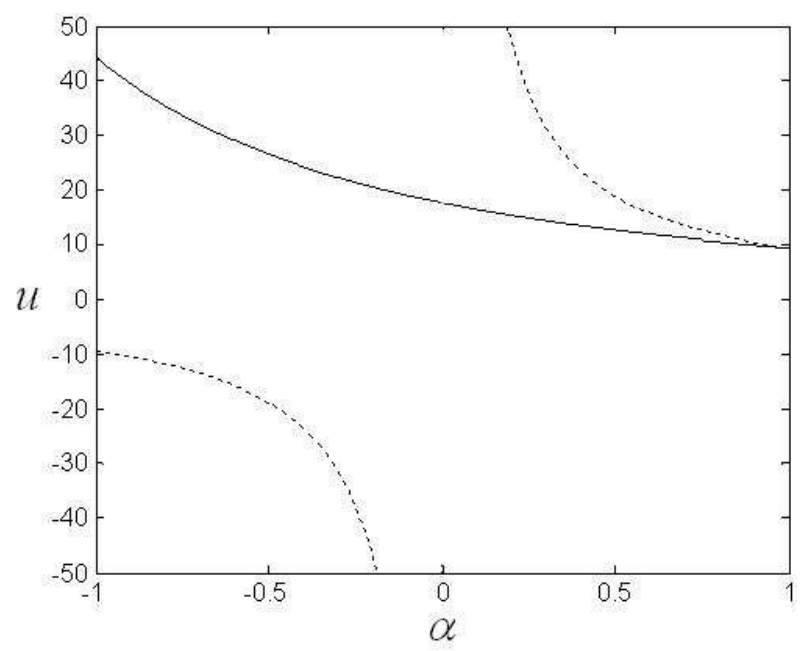

Figure 11. Comparison between the linearized solution and the existing solution as the nonlinear coefficient varies from -1 to 1 . [ $t=2, x=7.5$.

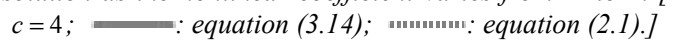


(i) For the existing soliton solutions, the parameter $\alpha$ only describes the steepening of the solitary wave. Unfortunately, the solitary wave will approach to infinity and become singular as $\alpha$ approaches zero, as shown in Figures 7-8. The reason the waves become singular is entirely due to their solution forms being proportional to $1 / \alpha$. This paradox has been overcome by the linearized soliton solutions. Figures 9-10 show that, under the same physical conditions, the linearized soliton solutions will not become infinity when $\alpha$ is zero. Instead, they will become linear exponential solutions. As the nonlinear term coefficient $\alpha$ varies from -1 to 1 , with $c=4, x=7.5$ and $t=2$, the difference between equations (2.1) and (3.14) can be demonstrated, as shown in Figure 11. It can be seen that the linearized solution is a continuous function of $\alpha$, while the existing solution has discontinuity at $\alpha=0$. It is noted that equations (3.14-15) are the same as equation (2.1) when $\alpha=1$. This assertion is demonstrated through Figures 7 and 9-11.

(ii) (In Figures 7-8, the steep slope of the existing soliton solutions are maintained in the same location; however, this is not the case for the linearized soliton solutions. Moreover, the location of the steep slope of the linearized soliton solutions will slide and change as $\alpha$ is changed. As shown in Figures 9-10, the location of the steep slope of the linearized soliton solutions slide to the right/left as $\alpha$ is changed from 2 to 0.2 . We have named this peculiarity the soliton-sliding phenomenon.

\section{Conclusions}

In this paper, the simplest equation method was employed to solve an important evolution equation namely the $\mathrm{KdV}$ equation, and obtained linearized solutions. The proposed derived solutions can be successfully reduced to linearity, while the nonlinear term coefficient becomes zero. Two differences between the linearized soliton solutions and the existing soliton solutions were elaborated. As expected, the wave speed $c$ presents the same two properties for those soliton solutions, affecting the steepening of the solitary wave and moving the wave location. However, it is not the case for the nonlinear term coefficient $\alpha$. With constants $x, c$, and $t$, the existing solutions are proportional to $1 / \alpha$, and will become infinity as $\alpha$ approaches zero. Although the steep slope in the existing soliton solutions are maintained in the same location, under the same physical conditions, the linearized soliton solutions will not become infinity when $\alpha$ is zero. Moreover, the location of the steep slope in the linearized soliton solutions slide, which was termed the soliton-sliding phenomenon. The reason the linearized soliton solutions slide is entirely due to the influence of linearity. Extensions of the simplest equation method to study different nonlinear partial differential equations without linearized solutions are expected in future works.

\section{Authors' Contributions}

The authors have equal contributions to each part of this paper. All the authors read and approved the final manuscript.

\section{Acknowledgement}

It is gratefully acknowledged that this work was supported by the Ministry of Science and Technology of Taiwan, under grants MOST 103-2221-E-006-048 and MOST 103-2218-E-006-016.

\section{References}

[1] Bullough, RK, Caudrey, PJ. Solitons. New York: Springer-Verlag Berlin Heidelberg (1980)

[2] Korteweg, DJ, G. de Vries. On the change of form of long waves advancing in a rectangular channel, and a new type of long stationary waves. Phil. Mag. 39.5, 422-443(1895)

[3] Wazwaz, AM. Partial differential equations and solitary waves theory. The USA: Springer (2007)

[4] Jeffrey, A, Kakutani, T. Weak nonlinear dispersive waves: a discussion centered around the Korteweg-de Vries equation. SIAM Review. 14.4, 582-636 (1972)

[5] Soliman, AA. The modified extended tanh-function method for solving Burgers-type equations. Physica A 361, 394-404 (2006)

[6] Wazwaz, AM. The tanh method for travelling wave solutions of nonlinear equations. Applied Mathematics and Computation 154, 713-723 (2004)

[7] Wazwaz, AM. The tanh method and the sine-cosine methods for a reliable treatment of the modified equal width equation and its variants. Communications in Nonlinear Science and Numerical Simulation 11, 148-160 (2006)

[8] Hirota, R. The direct method in soliton theory. Cambridge, Cambridge University Press (2004)

[9] Hereman, W, Nuseir, A. Symbolic methods to construct exact solutions of nonlinear partial differential equations. Math. Comput. Simulation. 43, 13-27 (1997)

[10] Ebaid, A. Exact solitary wave for some nonlinear evolution equations via Exp-function method. Phys. Lett. A 364, 213-219 (2007)

[11] Liu, S, et al. Jacobi elliptic function expansion method and periodic wave solutions of nonlinear wave equations. Physics Letter A 289, 69-74 (2001)

[12] Shen, S, Pan, Z. A note on the Jacobi elliptic function expansion method. Physics Letter A 309, 143-148 (2003)

[13] Wang, M, Wang, Y, Yubin, Z. An auto-Backlund transformation and exact solutions to a generalized $\mathrm{KdV}$ equation with variable coefficients and their applications. Physics Letter A 303, 45-51 (2002)

[14] Kudryashov, NA. One method for finding exact solutions of nonlinear differential equations. Commun Nonlinear Sci Numer Simulat 17, 2248-2253 (2012) 
[15] Vitanov, NK. On modified method of simplest equation for obtaining exact and approximate solutions of nonlinear PDEs:The role of the simplest equation. Commun Nonlinear Sci Numer Simulat 16, 4215-4231 (2011)

[16] Kudryashov, NA. Seven common errors in finding exact solutions of nonlinear differential equations. Commun. Nonlinear Sci. Numer. Simulat. 14, 3507-3529 (2009)

[17] Vitanov, NK. Application of simplest equations of Bernoulli and Riccati kind for obtaining exact travelling-wave solutions for a class of PDEs with polynominal nonlinearity. Commun Nonlinear Sci Numer Simulat 15, 2050-2060 (2010)

[18] Kudryashov, NA. Modified method of simplest equation:Powerful tool for obtaining exact and approximate travelling-wave solutions of nonlinear PDEs. Commun Nonlinear Sci Numer Simulat 16, 1176-1185 (2011)

[19] Kudryashov, NA, Loguinova, NB. Extended simplest equation method for nonlinear differential equations. Applied Mathematics and Computation 205, 396-402 (2008)
[20] Mohamad, JA, Petkovic, MD, Biswas, A. Modified simple equation method for nonlinear evolution equations. Applied Mathematics and Computation 217, 869-877 (2010)

[21] Lu, D, Hong, B, Tian L. New solitary wave and periodic wave solutions for general types of $\mathrm{KdV}$ and $\mathrm{KdV}$-Burgers equations. Commun Nonlinear Sci Numer Simulat 14, 77-84 (2009)

[22] Wazzan, L. A modified tanh-coth method for solving the KdV and KdV-Burgers' equations. Commun Nonlinear Sci Numer Simulat 14, 443-450 (2009)

[23] Spiegel, MR. Mathematical handbook of formulas and tables. McGRAW-Hill; (1968)

[24] Wazwaz, AM. Two reliable methods for solving variants of the $\mathrm{KdV}$ equation with compact and noncompact structures. Chaos, Solitons and Fractals 28, 454-462 (2006) 